\title{
Effect of organic flux on the colossal dielectric constant of $\mathrm{CaCu} 3 \mathrm{Ti} 4 \mathrm{O} 12$ (CCTO)
}

\section{Vishnu Razdan, Abhishek Singh, Brad Arnold, Fow-Sen Choa, Lisa Kelly, et al.}

Vishnu Razdan, Abhishek Singh, Brad Arnold, Fow-Sen Choa, Lisa Kelly, N. B. Singh, "Effect of organic flux on the colossal dielectric constant of CaCu3Ti4O12 (CCTO)," Proc. SPIE 9493, Energy Harvesting and Storage: Materials, Devices, and Applications VI, 949308 (18 May 2015); doi: $10.1117 / 12.2177694$

SPIE Event: SPIE Sensing Technology + Applications, 2015, Baltimore, Maryland, United States 


\title{
Effect of organic flux on the colossal dielectric constant of $\mathrm{CaCu}_{3} \mathrm{Ti}_{4} \mathrm{O}_{12}$ (CCTO)
}

\author{
Vishnu Razdan*, Abhishek Singh+ Bradley Arnold, Fow-Sen Choa, Lisa Kelly and N. B. Singh! \\ Department of Chemistry and Biochemistry, University of Maryland, Baltimore County, 1000 \\ Hilltop Circe, Baltimore MD 21025 \\ + Present Address: Penn State University, State College, PA 16802 \\ *Present Address: Carnegie-Mellon University, Pittsburgh, PA 15212 \\ 'Corresponding Author. E-mail address: singhna@umbc.edu
}

\begin{abstract}
We have used low temperature organics to achieve orientation of the grains of $\mathrm{Ca}_{2 / 3} \mathrm{Cu}_{3} \mathrm{Ti}_{4} \mathrm{O}_{12}$ (CCTO) compound to increase the resistivity. During the past fifteen years CCTO has been studied extensively for its performance as a dielectric capacitor. We have synthesized and grown large grains of pure $\mathrm{Ca}_{2 / 3} \mathrm{Cu}_{3} \mathrm{Ti}_{4} \mathrm{O}_{12}$ and doped compound, and studied the dielectric constant and resistivity. The grains were aligned by using a naphthalene-camphor eutectic. CCTO was mixed in the organic melt and oriented by the directional solidification method. This material has different characteristics than pure processed CCTO material. The effect of solidification conditions and its effect on the morphology and the dielectric constant, resistivity and loss tan delta of pure and doped CCTO are described in this article.
\end{abstract}

\section{KEYWORDS}

Calcium copper titanate, organic, solidification, grains, annealing, dielectric, resistivity

\section{INTRODUCTION}

During the past fifteen years perovskites have been studied extensively [1-5] for their performance as a dielectric capacitor. Calcium copper titanate, $\mathrm{CaCu}_{3} \mathrm{Ti}_{4} \mathrm{O}_{12}$ (CCTO), was the first member to be recognized and has been extensively studied [1-5] for preparation of materials, effect of doping and its performance. Dielectric constant for CCTO has been reported in various ranges depending on the processing conditions. Researchers have reported value of dielectric constant greater than 100,000 for pure CCTO. This class of materials has attracted attention since these have a remarkable ability to undergo a series of cationic exchange reactions resulting corresponding isomorphs. The road block for its application as a practical dielectric material is its low resistivity and low break down voltage. In order to overcome this problem and to achieve high resistivity material a substitution and doping approach has been used extensively. If the valency of substituting ion is different from the host ion, substitution is limited. The substitution studies can be summarized as; (a) isovalent (b) heterovalent substitution and (c) valence compensated. As one can expect, the heterovalent substitution causes defected structures due to compensation of the extra charge resulting from this substitution. The case of the valence compensated substitution is very complex since the combination of ions at the host sub-lattice has to maintain total electrical charge neutrality in the crystal. We have observed [6] significant decrease in dielectric values when we used valence compensated approach. In addition we observed that some substituted materials affected the grain size significantly and required higher processing temperature. In this paper we report the effect of directional solidification of CCTO materials in low temperature organic matrix on the characteristics of CCTO.

\section{EXPERIMENTAL METHODS}

2.1 Materials Synthesis: We used using as supplied parent components $\mathrm{CaCO}_{3}, \mathrm{CuO}$ and $\mathrm{TiO}_{2}$ powder for the synthesis of the stoichiometric $\mathrm{CaCu}_{3} \mathrm{Ti}_{4} \mathrm{O}_{12}$ compound. The listed purity for these chemicals were listed as $99.99+\%$. Uniform size particles were prepared by using a wig-L-bug in a closed quartz ampoule. The particles sizes were in the range of 50 to $100 \mu \mathrm{m}$ size. Pellets of the stoichiometric mixture were prepared using a pressure in the range of 8000 to $9000 \mathrm{lb} / \mathrm{inch}^{2}$. We used a temperature range of 700 to $750 \mathrm{C}$ for sintering and $1000 \mathrm{C}$ for the grain

Energy Harvesting and Storage: Materials, Devices, and Applications VI, edited by Nibir K. Dhar, Achyut K. Dutta, Proc. of SPIE Vol. 9493, 949308 - @ 2015 SPIE · CCC code: 0277-786X/15/\$18 · doi: 10.1117/12.2177694 
growth. We used optical microscope and scanning electron microscope for determining the morphology at several stages of the processing.

2.2 Treatment with Organics: We used naphthalene -camphor organic system for the organic treatment. The composition was closed to the eutectic composition for soaking and directional freezing. This low temperature organic eutectic melt was used to orient the particles in a single orientation by the directional solidification.

2.3 Fabrication, Dielectric Constant, Capacitance and resistance measurement techniques: The processed pellets were cleaned and polished to achieve good quality polished surfaces on polishers with pads and using several solvents. Silver paste was used as preliminary electrode to determine the resistivity, capacitance and dielectric constant.

\section{RESULTS AND DISCUSSION}

The stoichiometric composition of $\mathrm{CaCu}_{3} \mathrm{Ti}_{4} \mathrm{O}_{12}$ was prepared by mixing the powder after milling the powder. The typical size of powder ranged between 50 to $100 \mu \mathrm{m}$ sizes.

Previously several processes to achieve large grains and high dielectric constant have been explored. It is widely reported that processing has profound effect on the dielectric constant. Despite intensive research in the area, a significant improvement in the resulting materials led to low resistivity materials. This study focused on alignment of grains using low melting organic melt. To achieve this goal the first step was the preparation of CCTO powder. The powder was pressed in into the form of pellet by applying a pressure greater than 8000psi (shown in Figure 1a). A prepared pellet is shown in Figure 1(b). This pellet was placed for coarsening at $700 \mathrm{C}$ for 3 hours followed by 70 hours at $900 \mathrm{C}$ for the grain growth. Figure 1(c) is a pellet after the grain growth. Typical sample sizes were in the range of $12 \mathrm{~mm}$ in diameter. This pellet was used for the organic treatment.

Naphthalene-camphor eutectic was prepared by weighing parent components and mixing by melt freeze method [7]. We used 3.512 grams of Naphthalene and 6.561 grams of Camphor for this study since eutectic is at approximately at Weight Percentage of $65 \%$ Camphor. The uniform mixture was prepared by slowly melting the naphthalene on top of the camphor in a water bath while gently stirring to create the eutectic. In order to avoid the evaporation, temperature was raised with a rate of $\mathbf{K} /$ minute to $80 \mathrm{C}$, closed to the melting temperature of naphthalene. Since the eutectic temperature is much lower $(32 \mathrm{C})$, both materials completely mixed below $80 \mathrm{C}$. The reacted two pellets of CCTO were finely ground into the powder and mixed with the organic material.

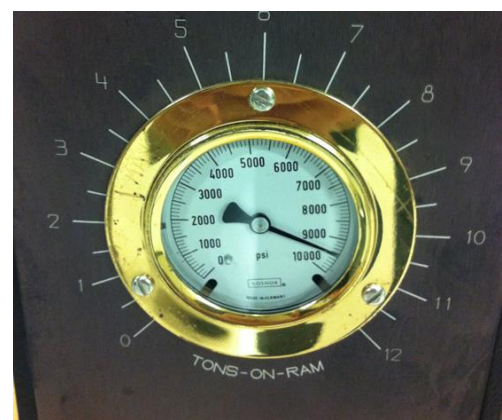

(a)

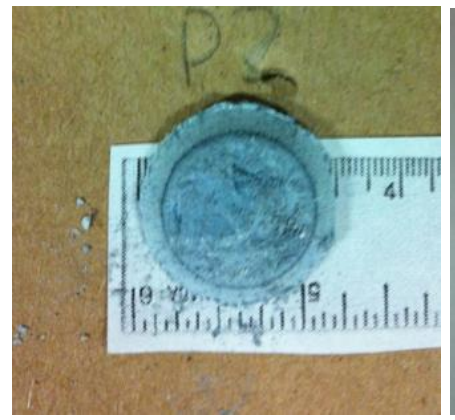

(b)

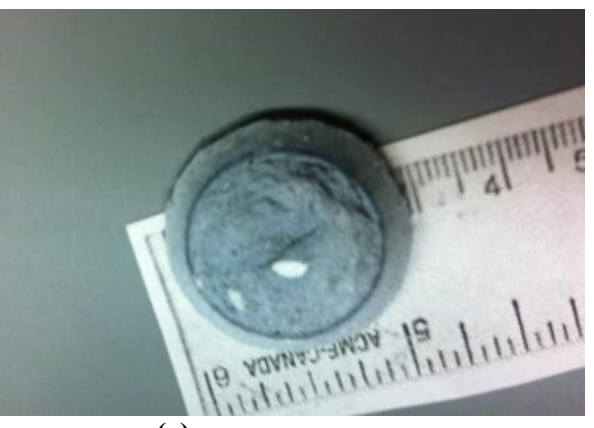

(c)

Figure 1.Pellet formation was performed at (a) a pressure $>8000 \mathrm{psi}$, (b) pre annealed and post annealed CCTO material

The organic eutectic melt is shown in Figure $2 \mathrm{a}$ without CCTO and in $2 \mathrm{~b}$ and $2 \mathrm{c}$ with CCTO powder. Figure $2 \mathrm{~b}$ shows a composite completely mixed with CCTO and Figure 2c shows composite where CCTO settled in the bottom of the melt. After shaking to ensure complete mixing in eutectic for soaking we used an ice cube to nucleate the composite in the bottom for directional freezing. The directional freezing in the eutectic melt was used to orient grains during the freezing. Figure $2 \mathrm{~d}$ shows the growth starting from the bottom of the container. The sizes of the containers were $13 \mathrm{~mm} \times 15 \mathrm{~mm}$ in diameter, and the length of the melt was $9 \mathrm{~cm}$. 


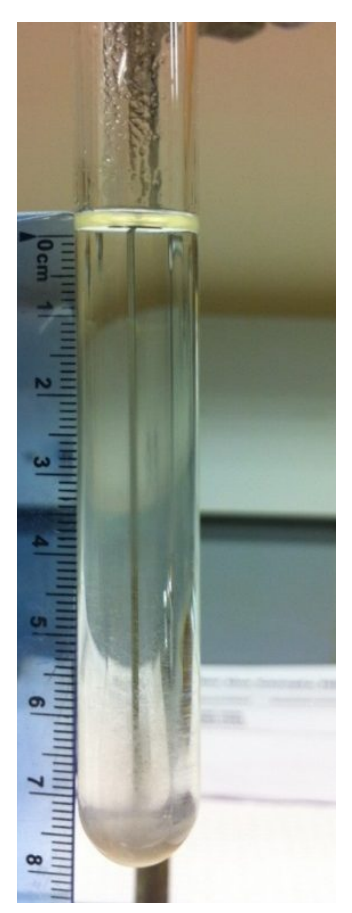

(a)

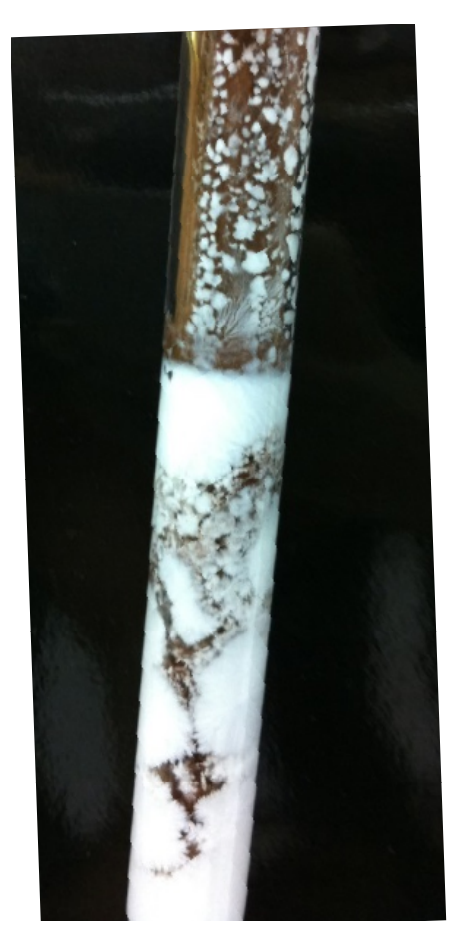

(b)

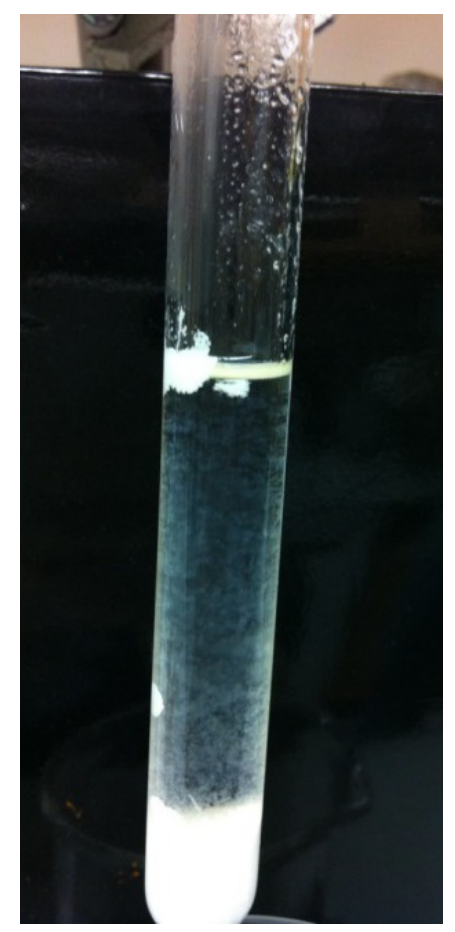

(c)

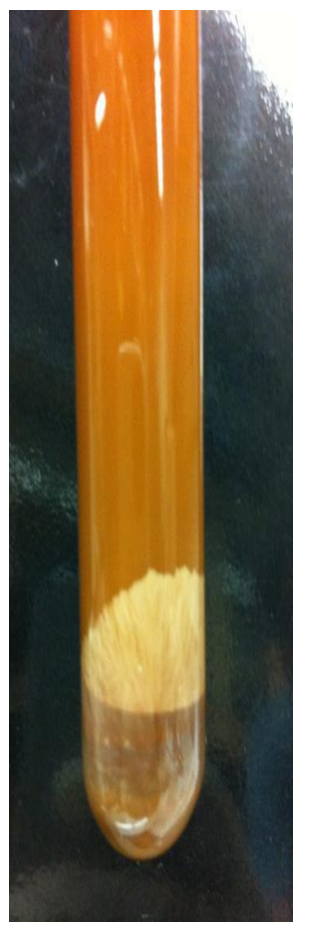

(d)

Figure 2. Using a water bath, the eutectic was heated to $60 \mathrm{C}$ to melt the eutectic fully, and then poured into another cylinder, where the eutectic undercooled.

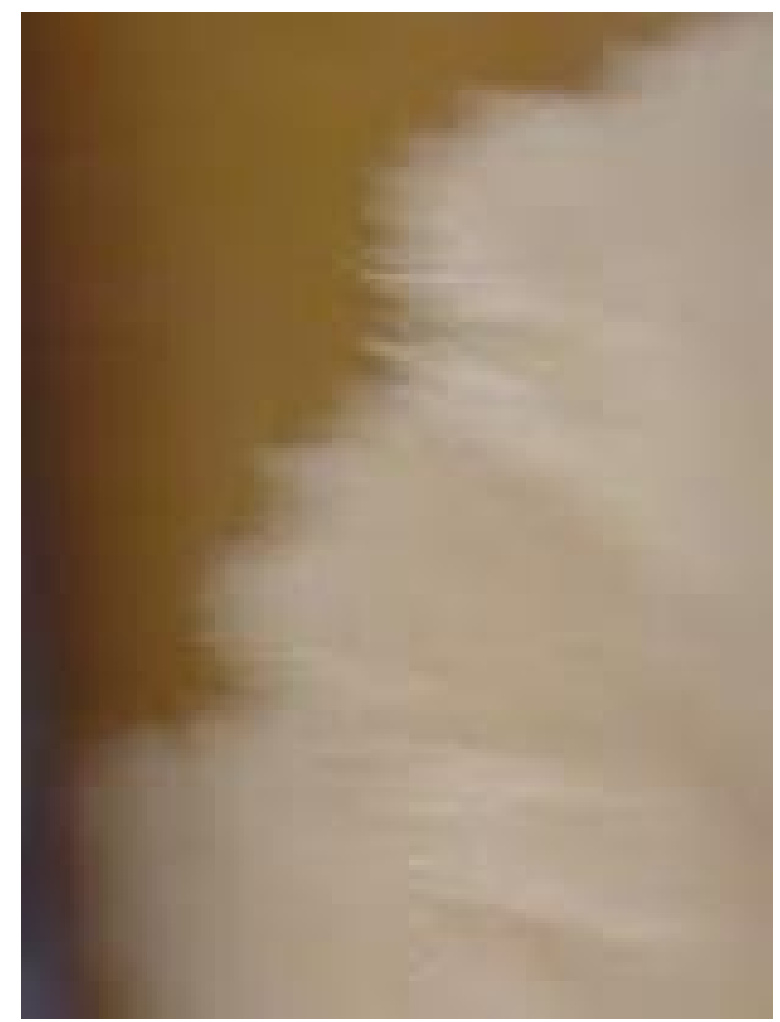

(a)

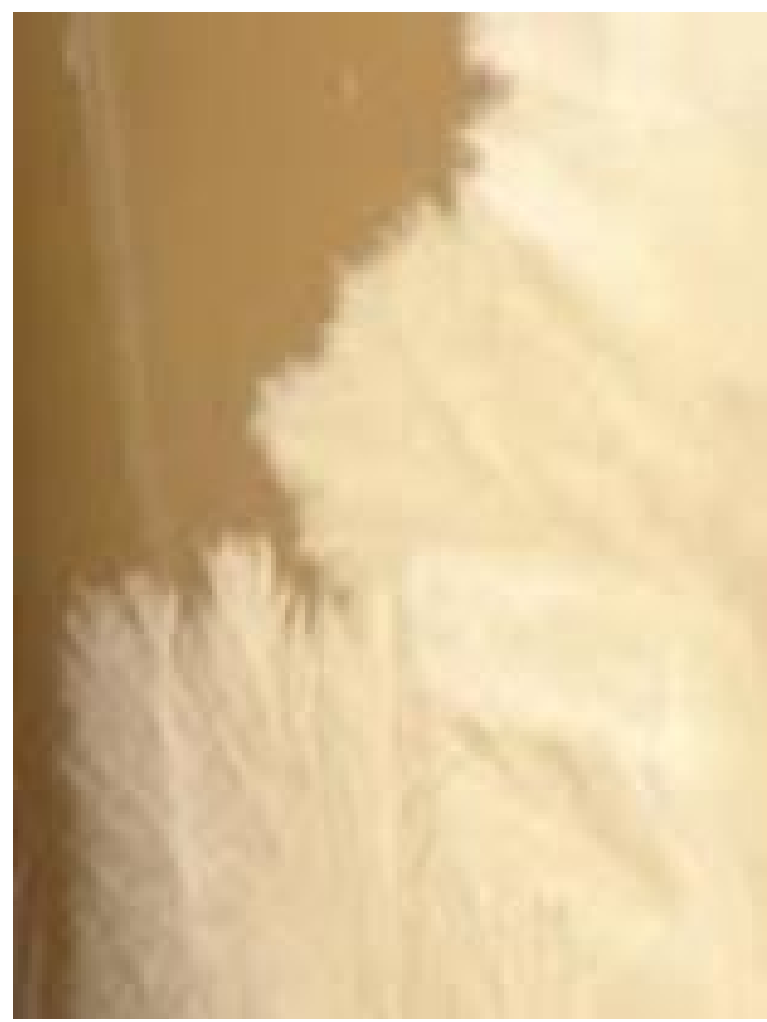

(b)

Figure 3 shows the microstructure of the material at 50x and 100x during the solidification 
The organic composite solidified in the dendritic morphology. This was very different than naphthalene-camphor eutectic morphology since CCTO significantly affected and modified the structure. We could even observed the dendrite tip splitting reported by Chopra, Singh and Glicksman [8]. The solidified material was placed in an oven at $50 \mathrm{C}$ for a period of 24-30 hours to remove organics. The resulting powder was again pressed into the form of a pellet and was further sintered. This material was used for the dielectric and resistivity measurement. The details of morphological evaluation indicated smaller grains compared to that of pure CCTO reported in reference 4. In addition we observed that identical processing of organic treated CCTO had unfilled spaces with high content of carbon. In addition, there were large numbers of smaller elongated grains and attached together in organic treated material where large grains with multiple orientations. Preliminary analysis showed presence of more liquid channels indicating that a higher temperature may be required for the processing of organic treated material.

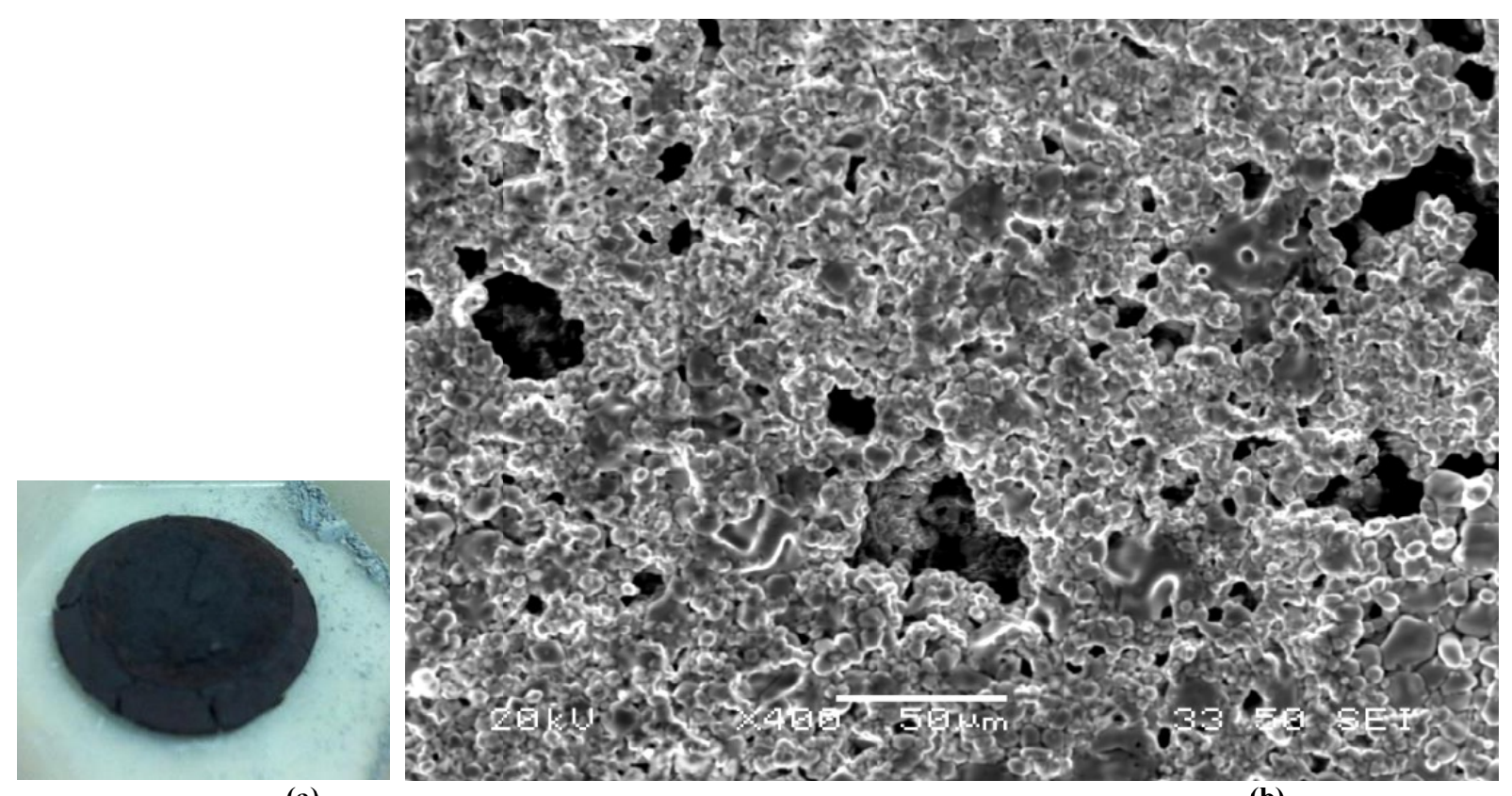

(a)

(b)

Figure 4. Organic treated CCTO (a) sample and (b) microstructure of the sample used for characterization. This sample also had some carbon impurity.

For the most of the dielectric and resistivity measurements we used silver pastes. However we tested with a gold electrode also. The material does not react with gold and long term stability is extremely good. Parallel-plate capacitors were fabricated from pressed and sintered pellets with thicknesses from 0.5 to $2 \mathrm{~mm}$ (thinned and polished). Electrodes were made with $\mathrm{Ti} / \mathrm{Au}$ thin film bilayers or with silver paint. We measured the dielectric constant in the range of 100 to $100,000 \mathrm{~Hz}$ frequency region. Results are shown in Figure 5. The dielectric constant for $100 \mathrm{~Hz}$ were observed to be in the range of $>33,000$, a value lower than that of pure CCTO values reported by Singh et al [4]. However, the resistivity for these samples were in the range of high $10^{8}$ to lower $10^{9} \mathrm{ohm}$-cm values at least two orders of magnitude higher than measured for the pure CCTO material. Further studies are required to identify the mechanism. 


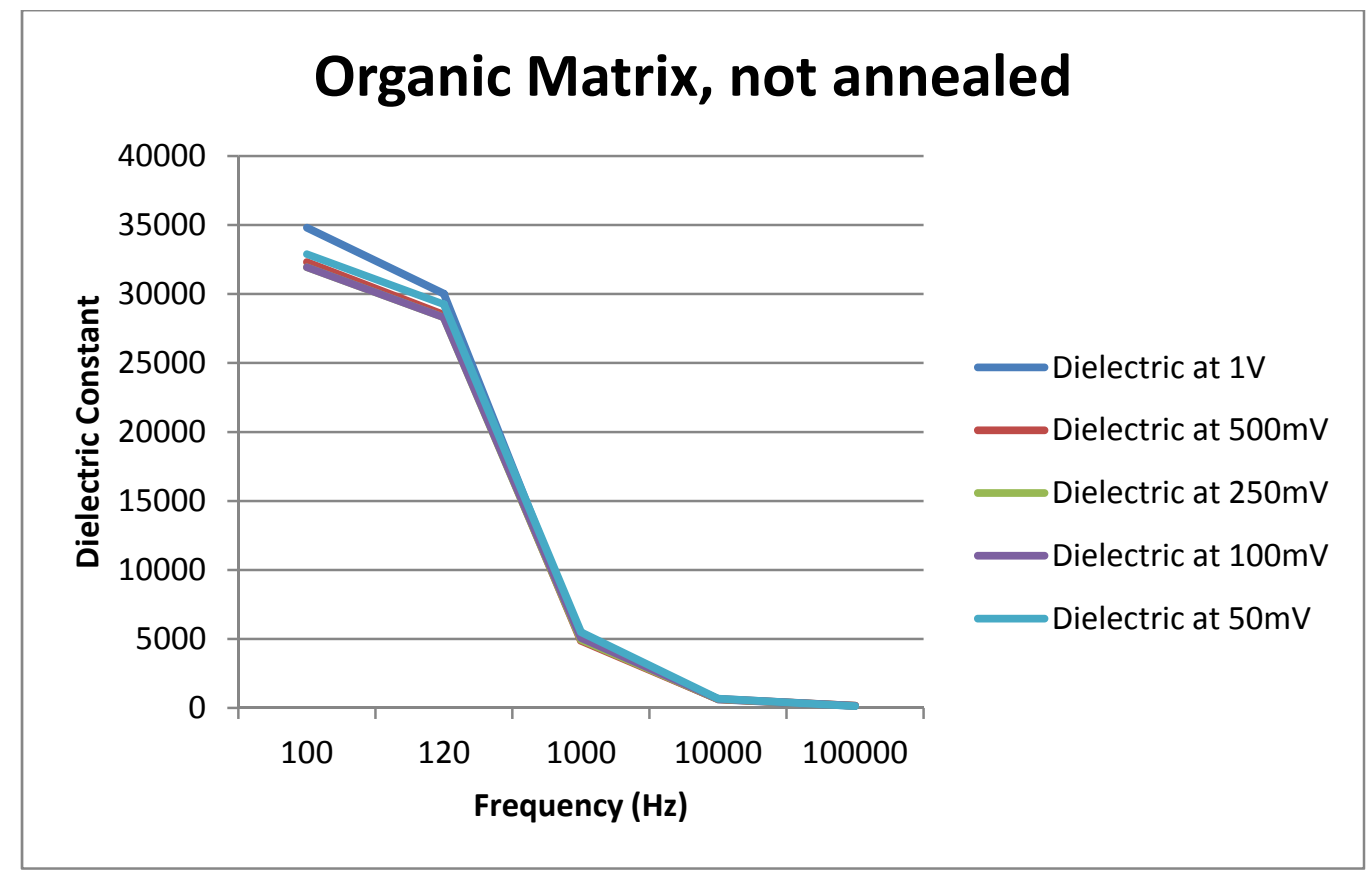

Figure 5. Dielectric values as function of frequency

\section{SUMMARY}

The $\mathrm{CaCu}_{3} \mathrm{Ti}_{4} \mathrm{O}_{12}$ (CCTO) powder treated with naphthalene-camphor eutectic melt and charge was directionally solidified. As synthesized and annealed CCTO was treated in the organic melt and directionally solidified. Naphthalene-camphor mixture in the range of $65 \mathrm{wt} \%$ of camphor was used as the matrix of the organic melt. The composite was directionally solidified and CCTO was recovered by evaporating the organics. Measured values were two orders of magnitude higher compared to that measured values in the range of $10^{5}$ to $10^{6} \mathrm{ohm}-\mathrm{cm}$ range for CCTO. Further research is continuing to clarify if oriented grains are cause of the high resistivity

\section{REFERENCES}

[1]. Subramanian, M. A., Dong, L., Duanand, N., Reisner, B.A., "High dielectric in $\mathrm{ACu}_{3} \mathrm{Ti}_{4} \mathrm{O}_{12}$ and $\mathrm{ACu}_{3} \mathrm{Ti}_{3} \mathrm{FeO}_{12}$ phases"J.Solid State Chem., Vol. 151, 323-325 (2000).

[2]. Singh, L., Rai, U.S., and Mandal, K.D., "Dielectric, modulus and impedance spectroscopic studies of nanostructured $\mathrm{CaCu}_{2.70} \mathrm{Mg}_{0.30} \mathrm{Ti}_{4} \mathrm{O}_{12}$ electro-ceramic synthesized by modified sol-gel route", Journal of Alloys and Compounds, Vol. 555, 176-183 (2013).

[3]. Cai, J., Lin, Y.H., Cheng, B., Nan, C.W., He, J., Yu, Y. and Chen, X., "Dielectric and nonlinear electrical behavior observed in Mn-doped $\mathrm{CaCu}_{3} \mathrm{Ti}_{4} \mathrm{O}_{12}$ ceramic" Appl. Phys. Lett., Vol. 91, 252905-3 (2007).

[4]. Singh, N. B., Berghmans, A., Knuteson, D., Talvacchio, J., Kahler, D., House, M., Schreib, B., Wagner, B., and King, M., "Evolution of microstructure due to additives and processing", J.Ceramic Transaction, 235, 65-76 (2012).

[5]. Singh, L., Rai, U.S., and Mandal, K.D., and Singh, N. B., "Progress in perovskite functional dielectric material $\mathrm{CaCu}_{3} \mathrm{Ti}_{4} \mathrm{O}_{12}$ ", J. Progress in Crystal Growth and Characterization, 60, 15-62 (2014).

[6]. Rai, U.S., Singh, L., and Mandal, K.D., and Singh, N. B., " An Overview on Recent Developments in the Synthesis, Characterization and Properties of High Dielectric Constant Calcium Copper Titanate Nano-Particles" Journal of Nanoscience and Technology, 1, (1)1-17 (2014).

[7] Singh, N. B. and Singh, Narsingh Bahadur, "Chemistry of Organic Eutectics", J. Crystal Growth, 28, 267-270 (1975).

[8]. Chopra, M., Singh, N. B. and Glicksman, M. E., Fundamentals of dendritic growth in alloys I", Met. Trans. A, 19A, 3087-93 (1987). 